\title{
Three-dimensional crustal density distribution beneath North China by sequential inversion of local earthquake traveltimes and gravity anomaly*
}

\author{
Xinsheng Wang ${ }^{1,2, \uparrow}$ Jian Fang ${ }^{1}$ and Houtse $\mathrm{Hsu}^{1}$ \\ ${ }^{1}$ Key Laboratory of Dynamic Geodesy, Institute of Geodesy and Geophysics, \\ Chinese Academy of Sciences, Wuhan 430077, China \\ ${ }^{2}$ Graduate University of the Chinese Academy of Sciences, Beijing 100049, China
}

\begin{abstract}
A three-dimensional crustal density model beneath North China is determined using P-wave traveltimes and gravity datum by sequential inversion method in this paper. To improve the uniqueness of the solution, we used a linear relationship between velocity and density to achieve mutual conversions and constraints between velocity difference and density difference. Algebraic reconstruction technique (ART) was used in density inversion, which highly improved the calculation speed comparing with common least squares method. The inversion results indicate that the crustal density beneath North China is extremely inhomogeneous with its distribution coherent with regional tectonics. The lower crust of Taihang mountain tectonic zone shows an obvious low density characteristic. We proposed that it may be an indicator of upwelling of mantle materials or partial melting of lowermost crust.
\end{abstract}

Key words: density distribution; sequential inversion; ART; seismic tomography CLC number: P315.2 Document code: A

\section{Introduction}

North China Craton has been a active area for the research on craton reconstruction all over the world (Wu et al., 2008; Zheng and Wu, 2009). Presently, the research on North China craton mainly focuses on the spatio-temporal distribution and mechanism of its destruction. Lithosphere destruction can lead to asthenosphere upwelling, sometimes partial melting, and further induce a series of geologic processes in the crust, such as magmatism, metamorphism, etc. To some extent, magmatism in crust can be used to confirm the lithosphere destruction. Therefore, the research on North China crust structure is necessary, which contributes to the research on shallow effects of craton

\footnotetext{
* Received 10 September 2010; accepted in revised form 9 January 2011; published 10 April 2011.

^ Corresponding author. e-mail: wxinsh@163.com

(C) The Seismological Society of China and Springer-Verlag Berlin Heidelberg 2011
}

destruction and further to research on lithosphere evolution and thinning, moreover, the destruction mechanism and spatio-temporal distribution of North China Craton. Density distribution can effectively reflect movement and variation of underground materials.

There are many results about crust structure of North China (Fang, 1999; Liu et al., 2003; Jia and Zhang, 2005; Tian, 2008). Bouguer gravity anomaly is sensitive to mass lateral variations, but it decreases in amplitude with the source depth increasing. Gravity inversion is a method with high resolution, but with a strong non-uniqueness for the solution. Seismic tomography can get an accurate three-dimensional velocity structure, but its resolution and accuracy strongly depend on the intersections and distributions of seismic rays, especially in a near-surface region for the nearly vertical rays.

We combined gravity anomaly and seismic travel time data by converting the density difference and velocity difference with Birch's law (Birch, 1961). Then, 
three-dimensional crustal density distributions of North China were determined by using sequential inversion method. Figure 1 shows the study area and its major geological tectonics. Gravity and tomography methods provide complement and constrain to each other, improving not only the resolution but also the uniqueness of the solution. The rationality and advantage of sequential inversion comparing with single inversion can refer to Vernant et al. (2002) in detail.

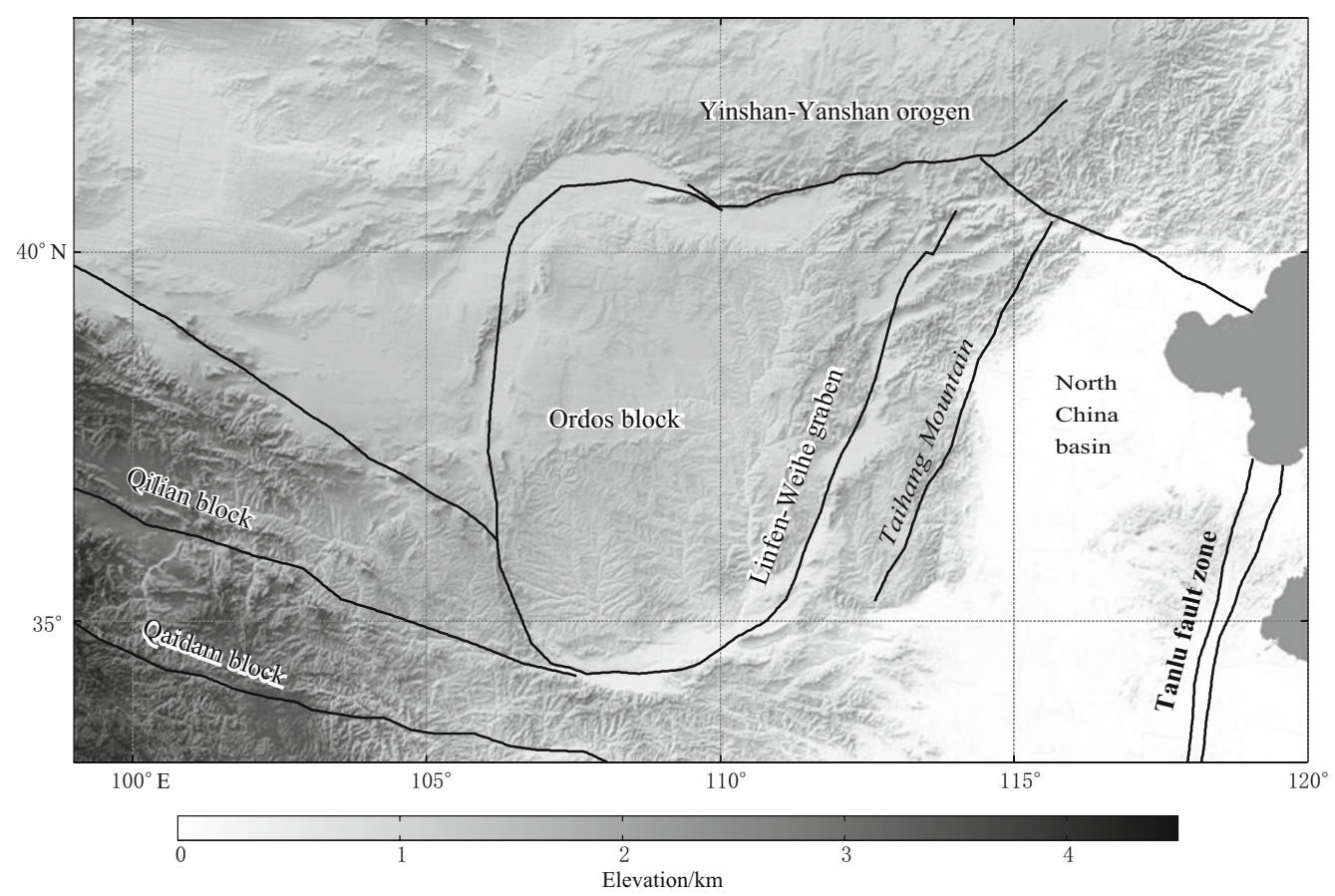

Figure 1 Major geological tectonics of the study area and its surrounding regions.

\section{Data and method}

\subsection{Data}

Two data sets were collected and combined to conduct sequential inversion in this study. One is arrival times from local and regional earthquakes which occurred in and around the North China region, and the other is the free-air gravity anomaly and interface undulation data of the research region. Totally, we use 149054 P-wave traveltimes from 7940 local and regional events recorded by 585 seismic stations during the period of 1980-2005 with the picking accuracy 0.1-0.2 s for most data in seismic tomography. In gravity inversion, we have collected $5^{\prime} \times 5^{\prime}$ free-air gravity anomaly to calculate Bouguer gravity anomaly after terrain correction and Bouguer correction. In the meantime, $30^{\prime \prime} \times 30^{\prime \prime}$ topography gridded data are used for terrain correction, and $30^{\prime} \times 30^{\prime}$ Moho and deposit interface undulation data are utilized to calculate their gravity contributions.

\subsection{Method}

The purpose of joint inversion of gravity data and seismic traveltime is to drive a crustal density and velocity model that account for both types of observations. At present, there are two main joint inversion ways: generalized joint inversion and sequential inversion. The former merges the two types of observation data into one equation where all data are inverted simultaneously. As the two data sets have different dimensions and resolutions, the relative weight is difficult to determine. Whereas the latter carries out gravity and seismic inversion alternatively until one model can simultaneously satisfy both the gravity anomaly and seismic traveltime data within acceptable misfits, thus avoiding determining the relative weight. As a result, sequential inversion is used in our study.

The a posteriori information resulting from the previous inversion of the first data set is transformed into a priori information to invert the second data set (Vernant et al., 2002). Figure 2 shows the organization of the sequential strategy. Firstly, we do the seismic inversion by using $\mathrm{P}$-wave traveltime, leading to a new three-dimensional velocity model and new event locations. Then, the velocity model mentioned above is converted to three-dimensional density model using Birch's 
law (Birch, 1961) as follows:

$$
v_{\mathrm{P}} \approx[3.31 \rho+f(w)] \pm 0.28,
$$

where $v_{\mathrm{P}}(\mathrm{km} / \mathrm{s})$ and $\rho\left(\mathrm{g} / \mathrm{cm}^{3}\right)$ are velocity and density, respectively. $w$ is the mean atomic weight of the rock. As the slope of Birch's law is constant independently of the mean atomic weight used, the density contrast and velocity contrast do not depend on the mean atomic weight. Therefore, in this study, we ignore $f(w)$. Nextly, to carry out the inversion of gravity data and obtain a new density contrast model by taking the density contrast model above as the initial density model. Then, the new density model above is transformed into initial velocity model for the next tomography. This procedure is repeated until the convergent criterion is satisfied.

In seismic inversion, tomography method (Zhao and Kanamori, 1992; Zhao and Hasegawa, 1993) is used.

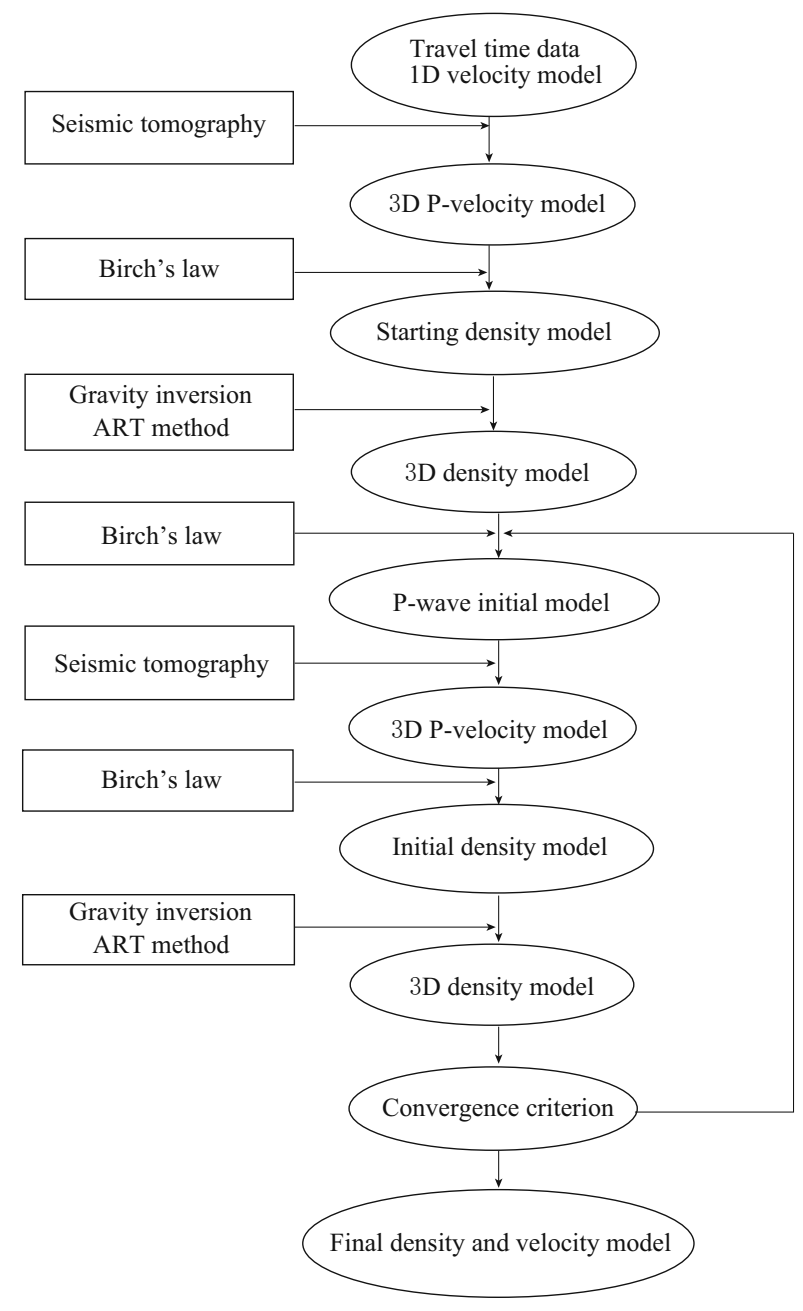

Figure 2 Flow-chart of the sequential inversion procedure used in this study.
This method can not only compute the travel times and raypaths accurately and rapidly, but also relocate the earthquake hypocenters in the inversion process. It can deal with a velocity model that contains complex velocity discontinuities, like the Moho discontinuity. In gravity inversion, the algebraic reconstruction technique (ART) is used, which is often called row action method. Here, the system of equations to be solved is not treated as a whole but single equation is considered at one time. Starting from an initial density value $d^{0}$, the elements of $d$ are iteratively modified through

$$
d_{j}^{k}=d_{j}^{k-1}+\frac{\lambda \cdot a_{i j}\left(g_{i}-\sum_{j=1}^{J} a_{i j} d_{j}^{k-1}\right)}{\sum_{j=1}^{J} a_{i j}^{2}},
$$

where $k$ and $\lambda$ are the iteration step number and relaxation parameter, respectively. $d$ is density, $g$ is observed gravity anomalies and $a_{i j}$ is the residual gravity field at the $i$-th point induced by the $j$-th cell with unit density, and depends on the distance between the calculation points and the gridded nodes. This method saves the internal storage of the computer and greatly improves the compute speed, for it avoids the matrix inversion of large and sparse system of observation equations by using row iterative technique.

\section{Inversion and results}

The gravity anomalies measured on the ground surface are the integrated reflection of the interface undulations and inhomogeneous density underground. Gravity anomalies resulting from Moho and deposit interface undulations are calculated using Parker's formula (Parker, 1972). Bowin (1983) gave the relationship between depth of the resource and spherical harmonics degree:

$$
Z=\frac{R}{n-1}
$$

where $Z$ is the depth of resource, $R$ is the Earth's radius and $n$ is the spherical harmonics degree. Long wave satellite gravity anomalies corresponding to 2-6 spherical harmonics degrees are obtained by EGM96 model. Finally, residual gravity anomalies resulting from crustal density inhomogeneous are formed after 2-6 order satellite gravity anomalies and gravity anomalies resulting from Moho and deposit interface undulations are deducted from Bouguer gravity anomalies.

We took $33^{\circ} \mathrm{N}-43^{\circ} \mathrm{N}, 99^{\circ} \mathrm{E}-120^{\circ} \mathrm{E}$ as our study area. Two kinds of grids are set up in this area for seismic inversion and gravity inversion, respectively. In 
depth, we set up six grid mesh layers at 5, 10, 20, 25, $30,35 \mathrm{~km}$ depths in the two grids. In the horizontal direction, for the fifth to the sixth layers the separation between grid nodes is $0.5^{\circ}$ in both grids. But for the first to the fourth layers, the separation is 0.5 in seismic inversion, while $0.25^{\circ}$ in gravity inversion.

Figure 3 shows the one-dimensional $\mathrm{P}$-wave velocity model which is used as the starting model for the first seismic inversion. The initial $1 \mathrm{D}$ velocity model is based on the previous research results (Zheng et al., 2007; Tian, 2008).

Then, seismic tomography gets a new 3D P-wave velocity contrast model and new earthquake locations from the arrival time of local events when the standard deviations between observed travel times and theoretical values satisfy the criterion. Figure 4 shows the result of seismic tomography. Figure 4 obviously illustrates a main low velocity anomaly characteristic beneath the southwest part of the study area above $30 \mathrm{~km}$, which is similar to the result given by Guan (2009). The Taihang

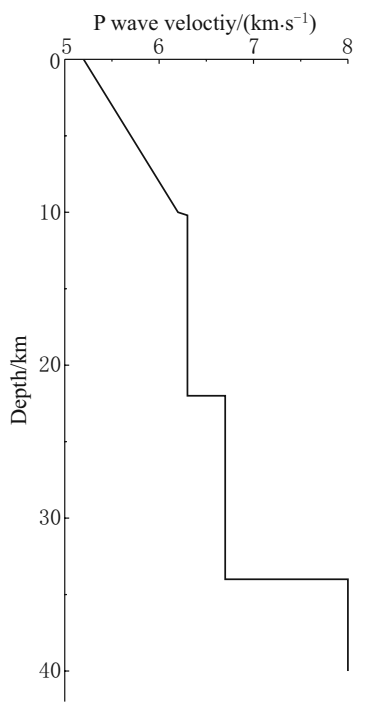

Figure 3 The initial 1D P-wave velocity model used in this study.
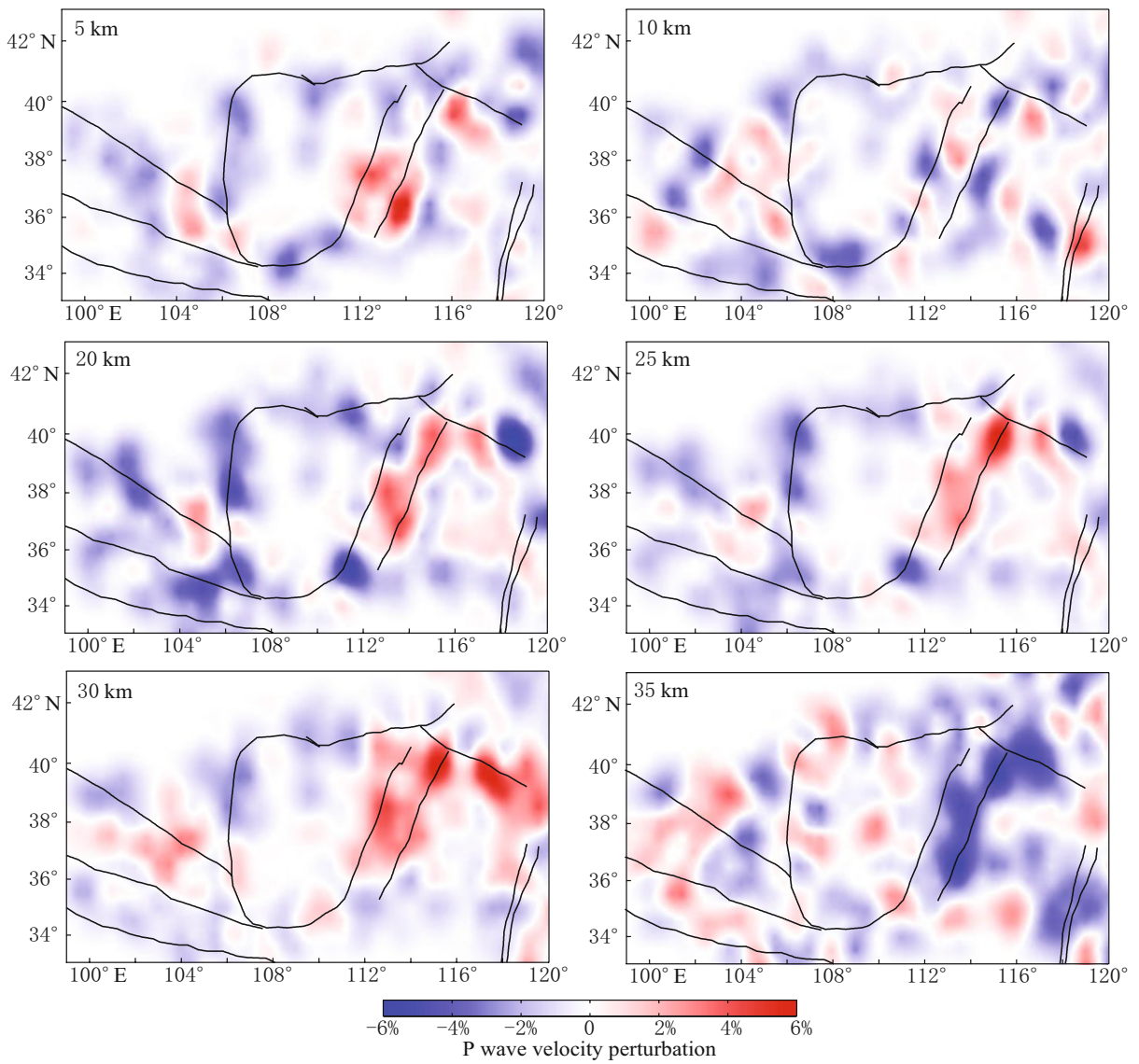

Figure 4 Map view of $\mathrm{P}$-wave velocity perturbation from the $1 \mathrm{D}$ velocity model as shown in Figure 3. The depth of each layer is shown at the upper-left corner of each map. Blue and red colors denote low and high velocities, respectively. The black lines are the tectonic lines. 
Mountain orogen is characterized by high velocity anomaly at $10-30 \mathrm{~km}$ depths. However, prominently low velocity distributes at $35 \mathrm{~km}$ depth of this orogen, which is consistent with the result of Tian et al. (2009). The resolution in Ordos block is not perfect, because of scant earthquakes in that area. So the velocity variation is relatively smooth.

The velocity contrast model above is then inverted into a 3D density contrast model using Birch's law. In the upper four layers, interpolation is needed. At this stage, ART is used to do gravity inversion, leading to a new density contrast model that is transformed back to a new velocity model for the next tomography. Then, velocity contrasts is inverted to density contrast as an initial density model again for the following gravity inversion. After this gravity inversion, we complete the first loop of the sequential inversion. Repeat the procedure until the standard deviations of the two data stop decreasing significantly between two loops of the sequential inversion. The final density model and the residual variances of gravity data versus the number of iterations are shown in Figures 5 and 6, respectively.
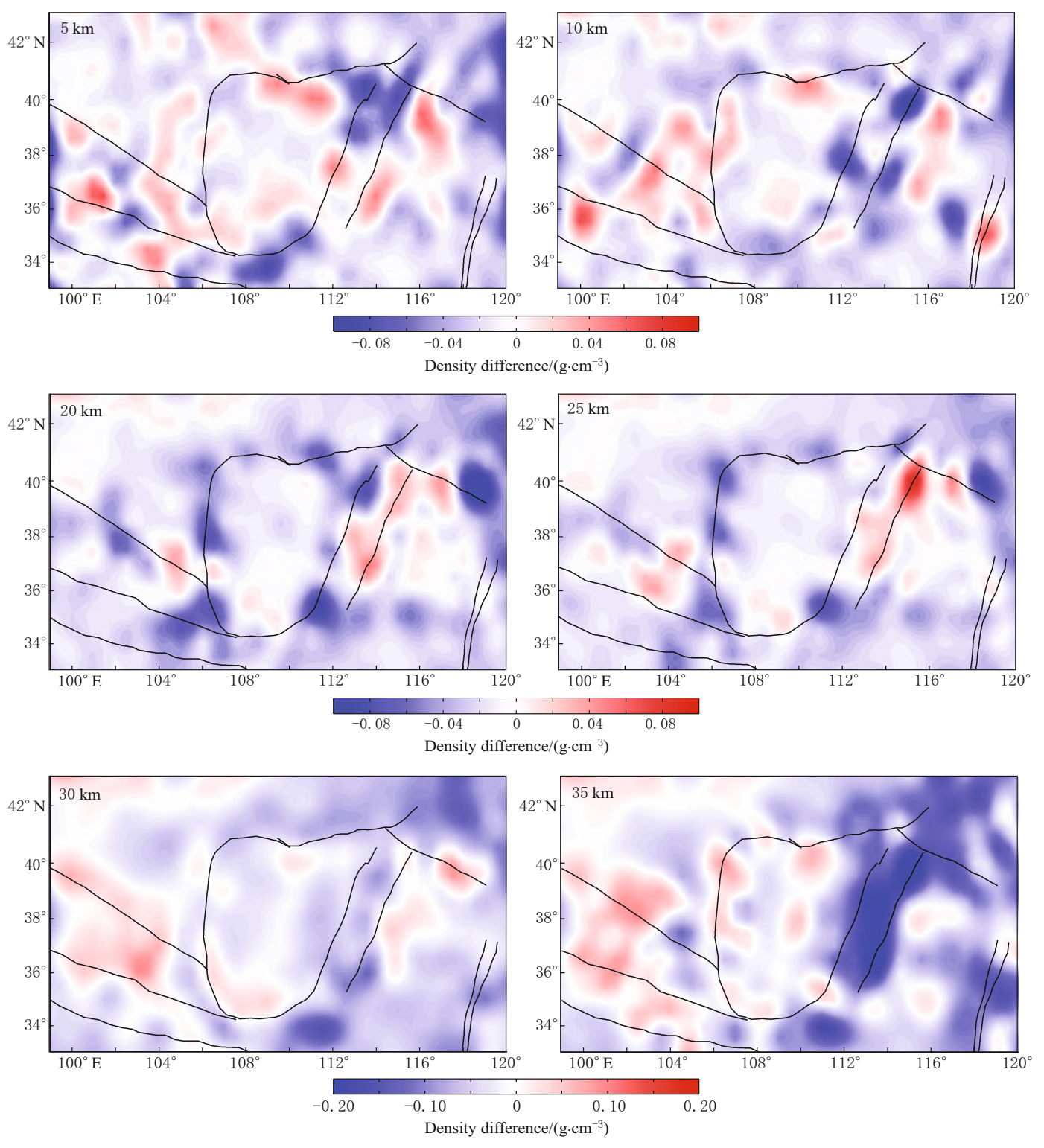

Figure 5 Map view of final density distribution. The depth of each layer is shown at the upper-left corner of each map. Blue and red colors denote low and high densities, respectively. The black lines are the tectonic lines. 


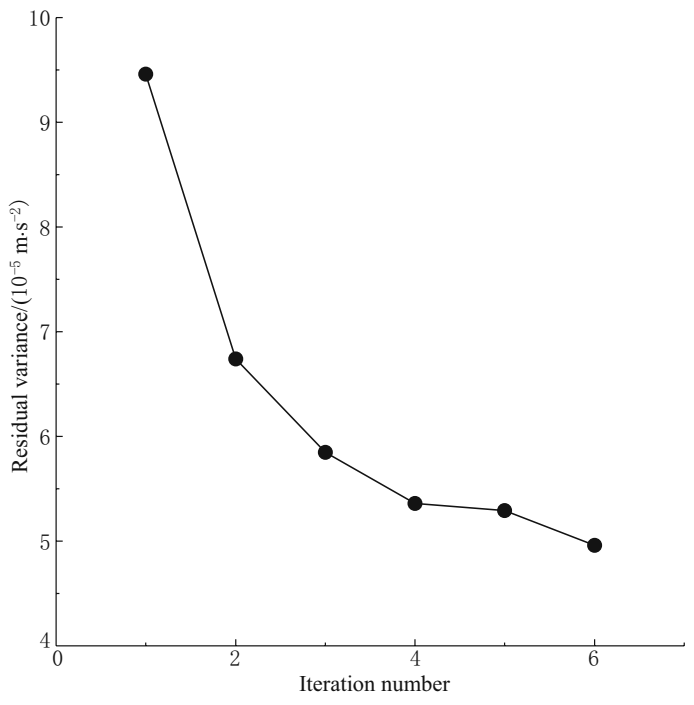

Figure 6 Residual variances of gravity data versus the number of iteration.

We can see from Figure 5 that crustal North China basin is characterized by high density anomaly, but with some exception in different depths. The upper and middle crust of the Tanlu fault zone exhibits a complex distribution of high and low density anomalies, and a low density anomaly with large amplitude exists at $35 \mathrm{~km}$ depth, however. The density contrasts are subdued in upper crust of Ordos block. At $30 \mathrm{~km}$ depth, high density distributes in southern part and northern part of the block while there is a prominently high density anomaly at $35 \mathrm{~km}$ depth.

A high density anomaly is visible beneath the Taihang Mountain orogen at $20-25 \mathrm{~km}$ depth. The density anomaly is high in the eastern part and is low in the western part at $30 \mathrm{~km}$ depth, while a strongly low density anomaly exists at $35 \mathrm{~km}$ depth, which may be the indication of mantle material upwelling or crust bottom partial melting. Qilian block has a complex distribution of low and high density anomalies down to $25 \mathrm{~km}$. Its lower crust exhibits a high density anomaly. As velocity and density of medium are affected by many factors, such as pressure and temperature, Birch's law is not consistent in some regions, which may bring errors to inversion results to some extent. We ignore these errors here.

\section{Conclusions}

In this paper, gravity anomaly and P-wave arrival time data are used to invert for the density structure in North China region. We obtain a $3-\mathrm{D}$ velocity model and density model of North China that can fit both types of observed data by sequential inversion. The crustal density beneath North China is extremely inhomogeneous, and it is obviously stronger in lower crust than in upper. The significantly low density anomaly beneath Taihang Mountain orogen at $35 \mathrm{~km}$ depth may be the indication of upwelling of mantle materials or partial melting of crust bottom. The algebraic reconstruction technique employed in gravity inversion improved the calculation efficiency tremendously comparing with the common least squares method. Our results provide a basic geophysical basis for further research of North China Craton destruction.

Acknowledgements We express our sincere thanks to professor Dapeng Zhao for providing his tomography code. We also appreciate Dr. You Tian and Jianshe Lei for their assistances on the study of the tomography code as well as two anonymous reviewers for their good advices.

\section{References}

Birch F (1961). Composition of the Earth's mantle. Geophys $J R$ astr Soc 4: 295-311.

Bowin C (1983). Depth of principal mass anomalies contributing to the earth's geoidal undulation and gravity anomalies. Marine Geodesy 21(7): 61-100.

Fang J (1999). Three-dimensional density distribution of lithosphere in North China. Geophysical and Geochemical Exploration 23(3): 179-184 (in Chinese with English abstract).

Guan J H (2009). Upper mantle structure of North China and surrounding regions from P-wave traveltime tomography. Progress in Geophysics 24(5): 1582-1 592 (in Chinese with English abstract).

Jia S X and Zhang X K (2005). Crustal structure and comparison of different tectonic blocks in North China. Chinese J Geophys 48(3): 611-620 (in Chinese with English abstract).

Liu Z P, Gao X L and Li Y S (2003). Density structure of the Taihang mountains gravity anomaly zone and its geological interpretation. Seismology and Geology 25(2): 266-273 (in Chinese with English abstract).

Parker R L (1972). The rapid calculation of potential anomalies. Geophys J R astr Soc 31(3): 447-455.

Tian Y (2008). Seismic Imaging Beneath the North China Craton and Southern California. Ph.D. Dissertation. Institute of Geology and Geophysics, Chinese Academy of Sciences, Beijing, 23 (in Chinese with English abstract).

Tian Y, Zhao D P, Sun R M and Teng J W (2009). Seismic imaging of the crust and upper mantle beneath the North China Craton. Physics Earth Planet Inter 172: 169-182. 
Vernant P, Masson F, Bayer R and Paul A (2002). Sequential inversion of local earthquake traveltimes and gravity anomaly - the example of the western Alps. Geophys $J$ Int 150: 79-90.

Wu F Y, Xu Y G, Gao S and Zheng J P (2008). Lithospheric thinning and destruction of the North China Craton. Acta Petrologica Sinica 24: 1145-1 174 (in Chinese with English abstract).

Zhao D and Hasegawa A (1993). P-wave tomographic imaging of the crust and upper mantle beneath the Japan Islands. J Geophys Res 98: 4333-4353.
Zhao D and Kanamori H (1992). P-wave image of the crust and uppermost mantle in southern California. Geophys Res Lett 19: 2329-2 332.

Zheng J C, Gu J P and Zhang Y S (2007). Combined inversion of the upper crust $3-\mathrm{D}$ velocity structure in north China. Progress in Geophysics 22(6): 1706-1714 (in Chinese with English abstract).

Zheng Y F and Wu F Y (2009). Growth and reworking of cratonic lithosphere. Chinese Science Bulletin 54(14): 1945-1949 (in Chinese with English abstract). 